\title{
UN TALLER ALFARERO EN EL BARRIO INDUSTRIAL URBANO DE GADES. A PROPÓSITO DEL HORNO CERÁMICO DE LA C/ SOLANO 3 (CÁDIZ).
}

\section{A POTTERY WORKSHOP IN THE URBAN INDUSTRIAL NEIGHBORHOOD OF GADES. ABOUT A CERAMIC KILN AT SOLANO 3 STREET (CÁDIZ)}

\author{
DARÍO BERNAL CASASOLA* \\ JOSÉ JUAN DÍAZ RODRÍGUEZ* \\ MARÍA LUISA LAVADO FLORIDO**
}

\begin{abstract}
Resumen: En estas páginas demos a conocer un avance del primer horno alfarero de época imperial localizado en el barrio industrial de la ciudad hispanorromana de Gades, resultado de una Actividad Arqueológica Preventiva en la C/ Solano. Se presentan tanto las características técnicas del horno como un avance de las cerámicas de manufactura local (ánforas y cerámicas comunes de diversa tipología).
\end{abstract}

Palabras clave: Gades, barrio industrial, alfar, horno, ánforas, cerámica común.

La pervivencia del hábitat en la ciudad de Cádiz desde su fundación en época fenicia hasta nuestros días ha provocado que en la actualidad exista una superposición de momentos históricos que dificulta la comprensión del urbanismo de épocas precedentes. Pese a ello, las distintas actuaciones arqueológicas que se han llevado a cabo en la ciudad en las últimas décadas han favorecido la interpretación de algunas áreas funcionales claramente diferenciadas del resto de espacios (Bernal 2008). En este sentido, la localización de algunas cetariae de época romana en el entorno del antiguo Canal Bahía - Caleta ha mostrado la existencia de un barrio industrial en dicho entorno urbano. Así, las factorías de salazón del Teatro Andalucía o del Cine Cómico en la

\footnotetext{
* Universidad de Cádiz.

** Arqueóloga
}

Abstract: In this paper we present a preliminary study of the first pottery kiln dating back to the I c. AD found inside the industrial quarter of the hispanorroman city of Gades. As a result of a Rescue Excavation at Solano street. We show the preliminary results of the study of the kiln as well as some details concerning the pottery produced locally (amphorae and plain pottery).

Key words: Gades, industrial area, pottery workshop, kiln, amphorae, common pottery.

ensenada septentrional del canal, la localizada en la $\mathrm{C} /$ Sagasta en la ensenada meridional del mismo o las de La Caleta y Castillo de Santa Catalina ubicadas en el reborde marítimo externo nos ofrecen una concentración de complejos dedicados a la explotación de los recursos marinos desde mediados del s. I a.C. hasta al menos el s. V-VI d.C. (Expósito 2007).

La aparición de un horno cerámico en la C/ Solano ha multiplicado exponencialmente la importancia de este discurso, pues ahora a la industria conservera hay que sumarle la existencia de actividades alfareras, lo que favorece aún más la interpretación de que estemos ante un barrio industrial intra moenia polifacetado de notables dimensiones; asimilable entonces a otros casos de ciudades atlánticas hispanorromanas como Olisipo o Baelo Claudia. Junto con el horno de la $\mathrm{C} /$ Solano, el primer horno romano documentado en 


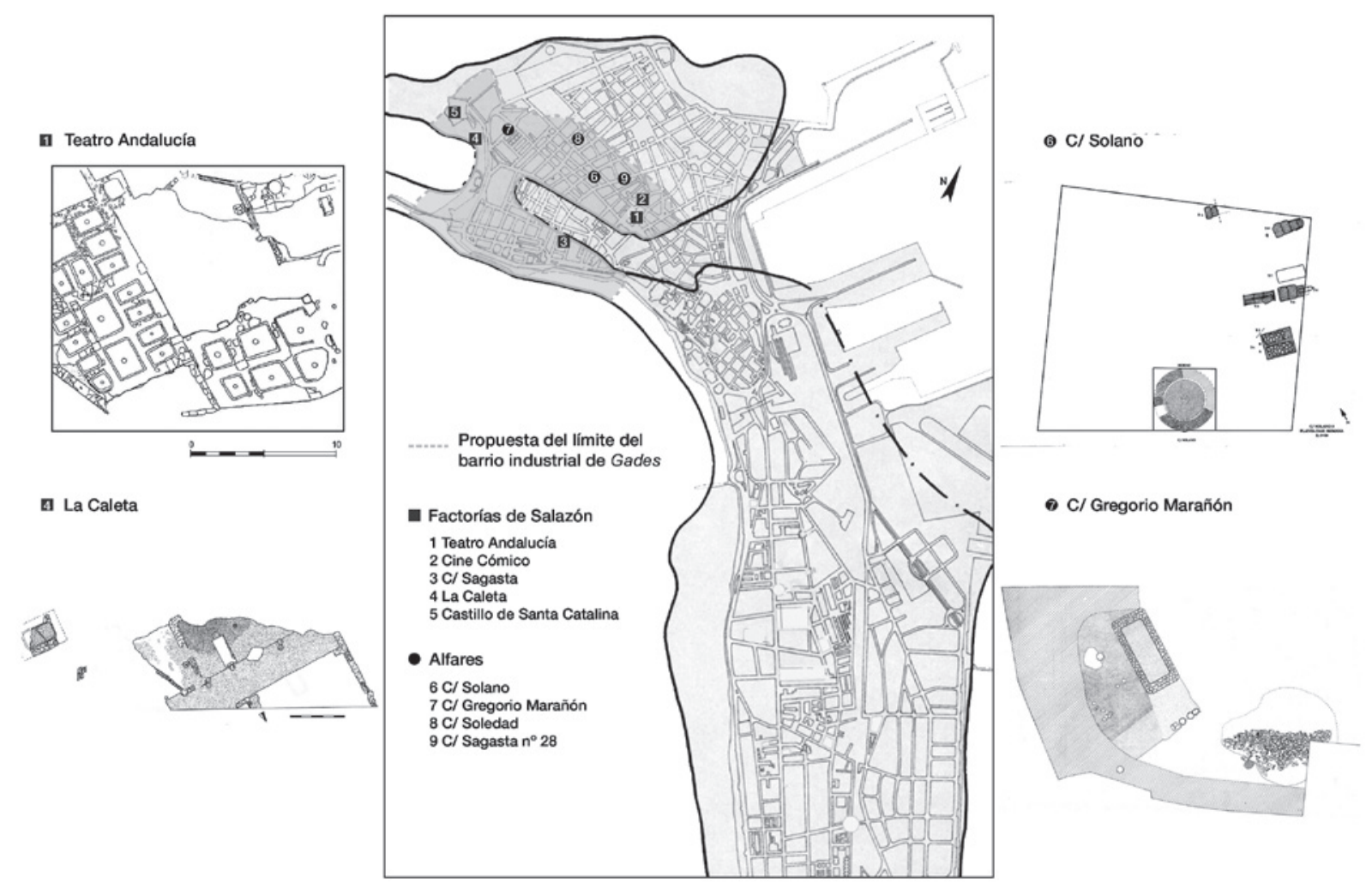

Figura 1. Plano de localización del barrio industrial de Gades sobre la topografía actual de la ciudad de Cádiz, con indicación de las factorías de salazón (cuadrado) y los alfares (círculo). En el lateral izquierdo la planta de la factoría de salazón del Teatro Andalucía y La Caleta. En lateral derecho planta del alfar de C/ Solano y de la posible figlina de Gregorio Marañón (elaboración propia a partir de original de L. Aguilera; Blanco 1991; Cobos, Muñoz y Perdigones 1997 y Expósito 2007: fig. 3 y 57).

el interior del perímetro urbano de Gades, existen también evidencias de producción alfarera en el entorno. De esta manera, en la C/ Gregorio Marañón se documentó una pileta que, aunque en un primer momento fuera interpretada como perteneciente a una factoría de salazón (Blanco 1991), bien podría tratarse de una cubeta para la decantación de arcillas (García Vargas 1998: 159). Junto a ésta se localizaron una serie de pavimentos, así como un vertedero anfórico, todo ello dependiente de una misma figlina. Por otro lado, en la $\mathrm{C} /$ Sagasta se han atestiguado algunos estratos de vertidos que contenían fallos de cocción de ánforas, cerámicas comunes e incluso sigillatas precoces o tipo Peñaflor (Blanco y Alarcón 1996). Por último, en la C/ Soledad se han localizado recientemente una serie de bolsadas de materiales cerámicos altoimperiales, en curso de estudio en la actualidad, entre las que destaca la existencia de diversos fallos de cocción de ánforas, lo que redunda en la idea de la proliferación de centros productivos concentrados en un mismo espacio. Algo también extensible a los numerosos defectos de cocción cerámicos procedentes de las recientes excavaciones en el antiguo Cine Cómico (Fig. 1).

Centrándonos en el análisis del alfar de la C/ Solano $\mathrm{n}^{\mathrm{o}} 3$, la intervención arqueológica deparó el hallazgo en el extremo meridional del solar de un horno altoimperial del tipo Ia de Cuomo di Caprio. Se trataba entonces de una estructura de planta circular con pilar central también circular, cuyo praefurnium se extendía más allá de los límites de la parcela, por lo que no pudo ser excavado de manera integral (Fig. 2). Del resto del complejo alfarero no se localizó ningún otro tipo de área funcional (zona de trabajo, vertederos, etc...), habiéndose documentado en el resto de la parcela un área de necrópolis de época tardorromana, así como el aljibe de la casa derribada, el cual afectó tanto a la fase altoimperial como a la tardorromana.

El horno conservaba aún la cámara de combustión, parte de la cámara de cocción, el praefurnium, así como el arranque del pilar central y restos de la parrilla 


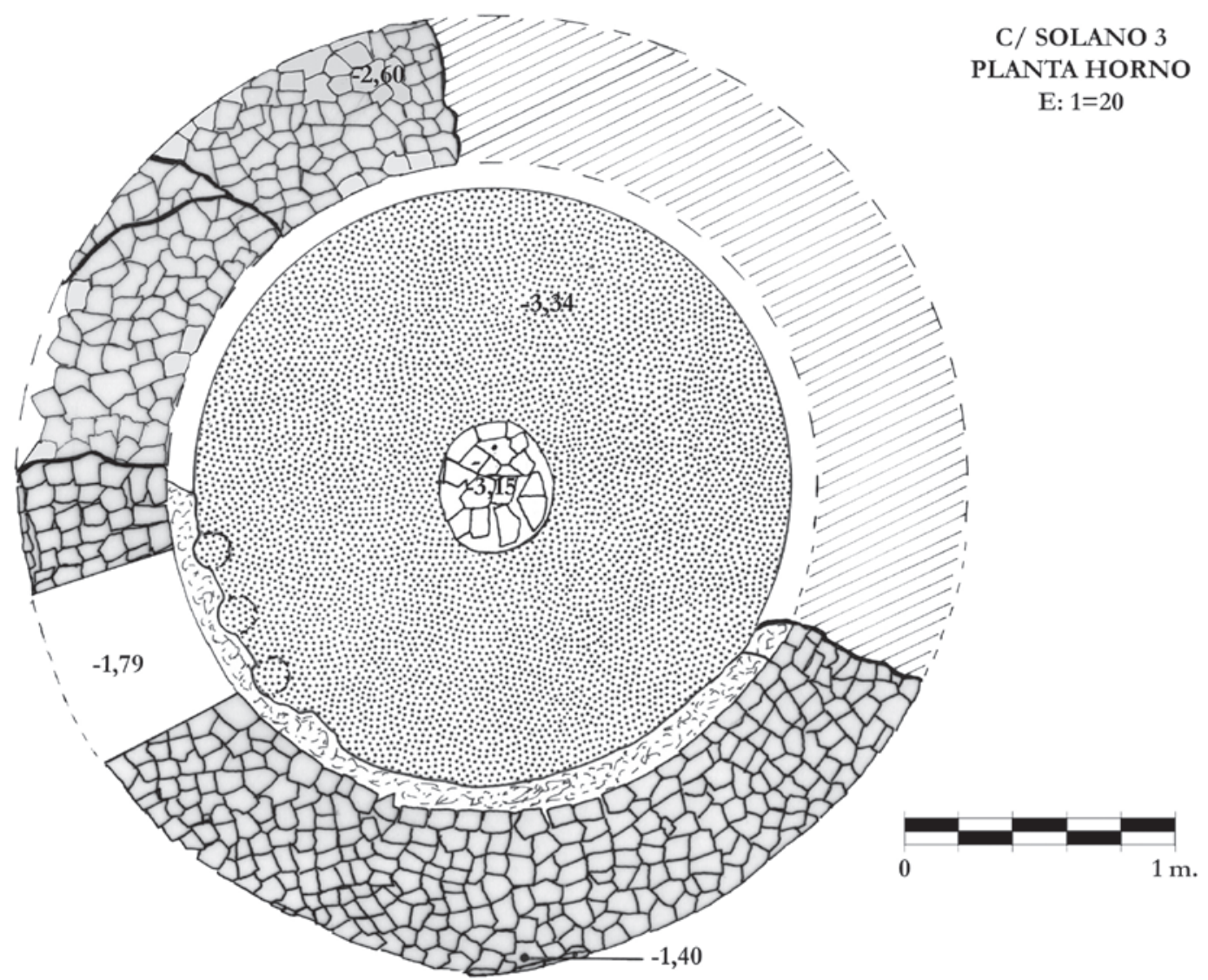

Figura 2. Planta del horno documentado en el alfar de la C/ Solano. Dibujo original de L. Aguilera.

(Fig. 3). Sin embargo, no toda la estructura se mantenía en el mismo estado de conservación. En su mitad nororiental el horno había sido alterado en momentos posteriores a su abandono, por lo que apenas conservaba restos de su alzado. En lo relativo a sus dimensiones, la planta del horno presentó un diámetro externo de 3,56 m. Por su parte, la cámara de combustión presentaba un alzado de $1,20 \mathrm{~m}$ y un diámetro interno de 2,21 m. En la zona central de esta cámara se levantó una columna realizada con material cerámico reutilizado, que constituyó el único elemento sustentante de la parrilla. Ésta, también de tendencia circular, alcanzó unas dimensiones de $50 \mathrm{~cm}$ de diámetro, conservando unos $40 \mathrm{~cm}$ de alzado. Este pilar debió sustentar la parrilla, de la cual apenas se conservaba la zona más próxima a la pared del horno. En este sentido, en la pared occidental se conservaron los restos de varias toberas -tres seguras y otras tantas posibles-, las cuales eran circulares y tenían entre 12 y $15 \mathrm{~cm}$ de diámetro. Para favorecer la sustentación de la parrilla y dado que la estructura no presentaba arcos que ayudaran al pilar central, la cámara de cocción era más ancha que la de combustión $\mathrm{y}$, por tanto presentaba un retranqueo de unos $20 \mathrm{~cm}$ de anchura que favorecía el arranque de la parrilla y su apoyo. Del praefurnium, orientado hacia el sur y configurado por una pseudo-bóveda de cañón, desconocemos su longitud total, pues se adentraba más allá de los límites físicos de la excavación. Pese a ello, se pudo excavar su conexión con la cámara de combustión. Alcanzó una altura máxima de $80 \mathrm{~cm}$ y una anchura máxima de $70 \mathrm{~cm}$. Por último, de la cámara de cocción se localizó un alzado de aproximadamente 70$80 \mathrm{~cm}$, desconociéndose por el momento si la estructura tuvo un laboratorium fijo o semipermanente. En su zona oriental, la cámara de cocción presentaba una discontinuidad en el alzado de su pared, la cual no podemos por el momento afirmar si se trata de una rotura o de un posible acceso a dicha cámara.

En cuanto al material empleado para su construcción, la piroestructura estaba construida mayoritariamente 


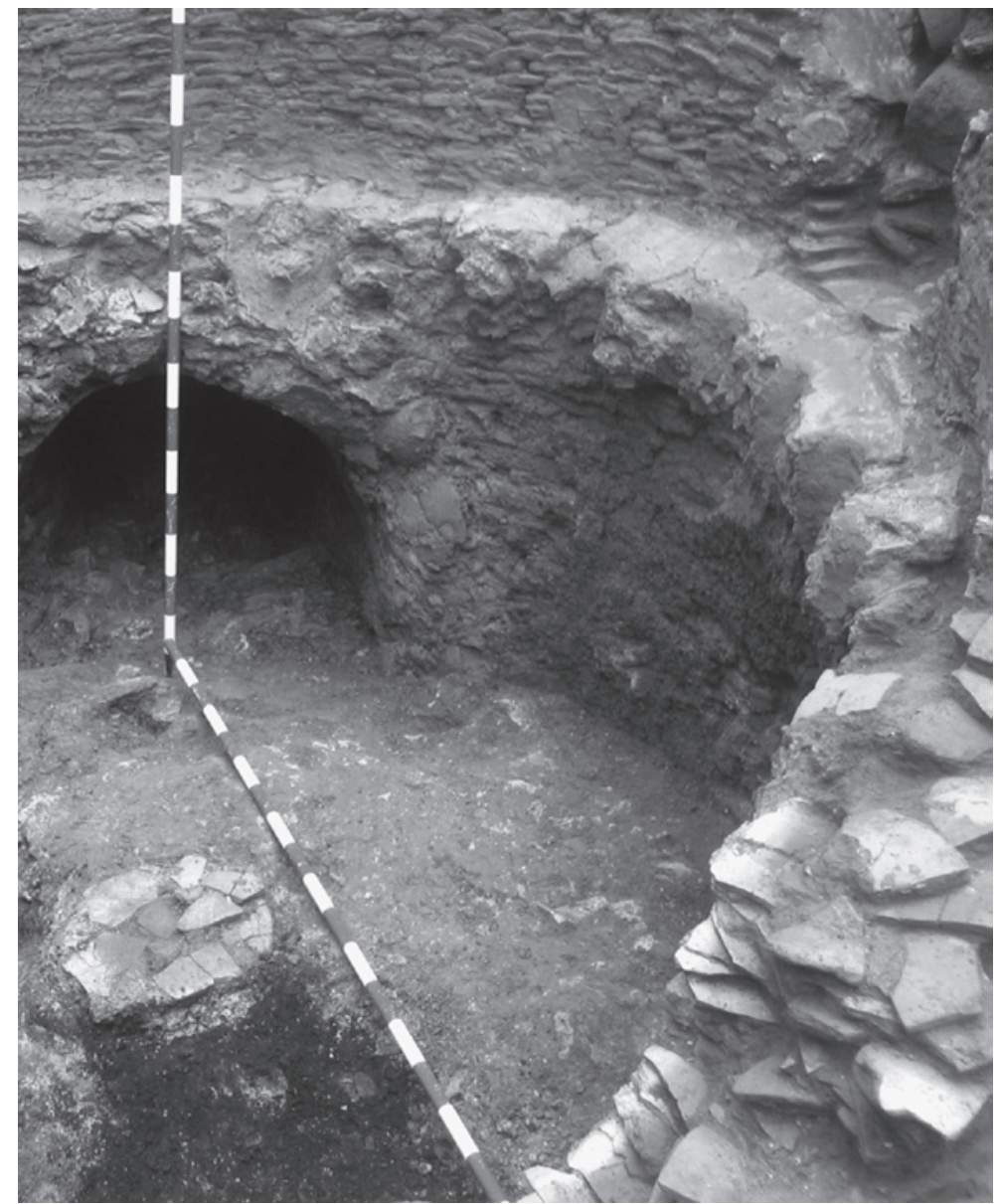

Figura 3.- Detalle del interior del horno de la C/ Solano. con material cerámico reutilizado -galbos anfóricos-, así como sillarejo pétreo de mediano tamaño, aglutinado todo ello con arcilla muy depurada y un fuerte grado de compactación. Sólo la parrilla estuvo realizada únicamente con arcilla y adobes. Para su construcción se tuvo que realizar una fosa en las arenas sedimentarias, que configuraban el sustrato edáfico del terreno en época romana en la margen septentrional interior del Canal Bahía - Caleta. La excavación del interior de la cámara de combustión y de parte del praefurnium deparó el hallazgo de una capa de unos $20 \mathrm{~cm}$ de potencia de cenizas muy compactadas y termoalteradas que evidenció el uso continuado de la piroestructura, habiéndose generado, entonces, un suelo de uso a una cota superior al primigenio, formado por una capa de arcilla.

Por último, en cuanto a la cronología del complejo alfarero, volvemos a insistir en la parcialidad del registro y en el estado incipiente de la investigación. Como dato de interés hay que decir que el horno estaba construido con galbos anfóricos. Este tipo de construcción es típico de estos ambientes en los que se reutiliza material cerámico desechado de otras hornadas para la construcción de nuevos hornos. Por tanto, existe una posibilidad de que en este complejo alfarero existieran estructuras de combustión precedentes a la documentada ahora. Por otro lado, los contextos excavados sólo nos permiten hablar del momento de abandono del horno, así como de la producción del mismo. En el relleno de la cámara de combustión se han documentado algunos fallos de cocción de ánforas salazoneras del tipo Dr. 7/11 y Beltrán IIa, así como posiblemente ánforas Dr. 12. Junto con el material anfórico en este alfar se habrían manufacturado diversas formas de cerámica común, entre las que destacan platos, jarras de amplia boca, estrecho y desarrollado cuello -con o sin filtro-, cuerpo globular y fondo plano con pie anular; así como opérculos y askoi rematados en pitorros con boca troncocónica y filtro interior, desconociéndose en el estado actual de la investigación en qué porcentaje se producirían las diversas categorías vasculares mencionadas -ánforas y cerámica común-. Finalmente, junto con el 


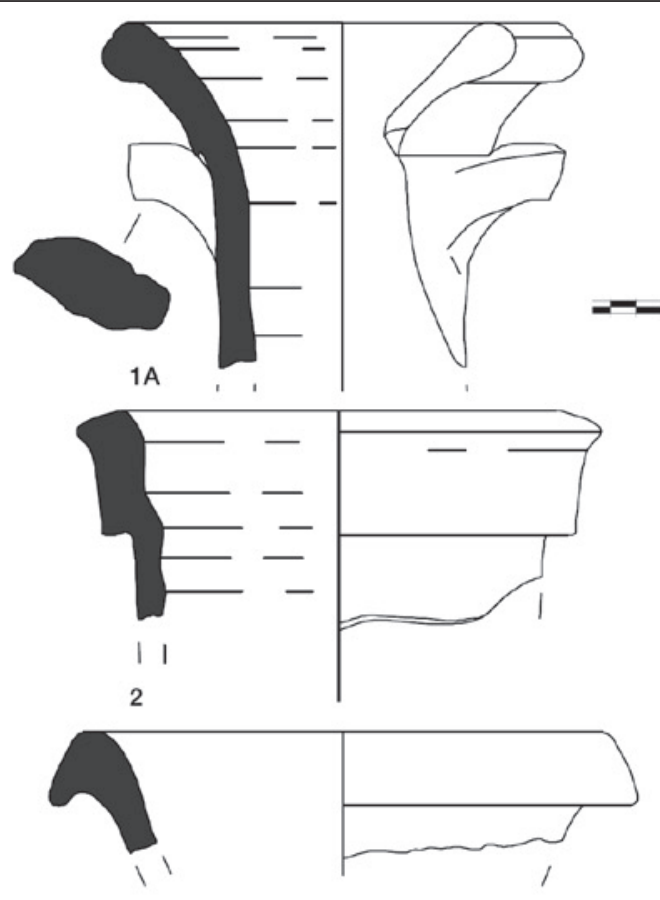

4
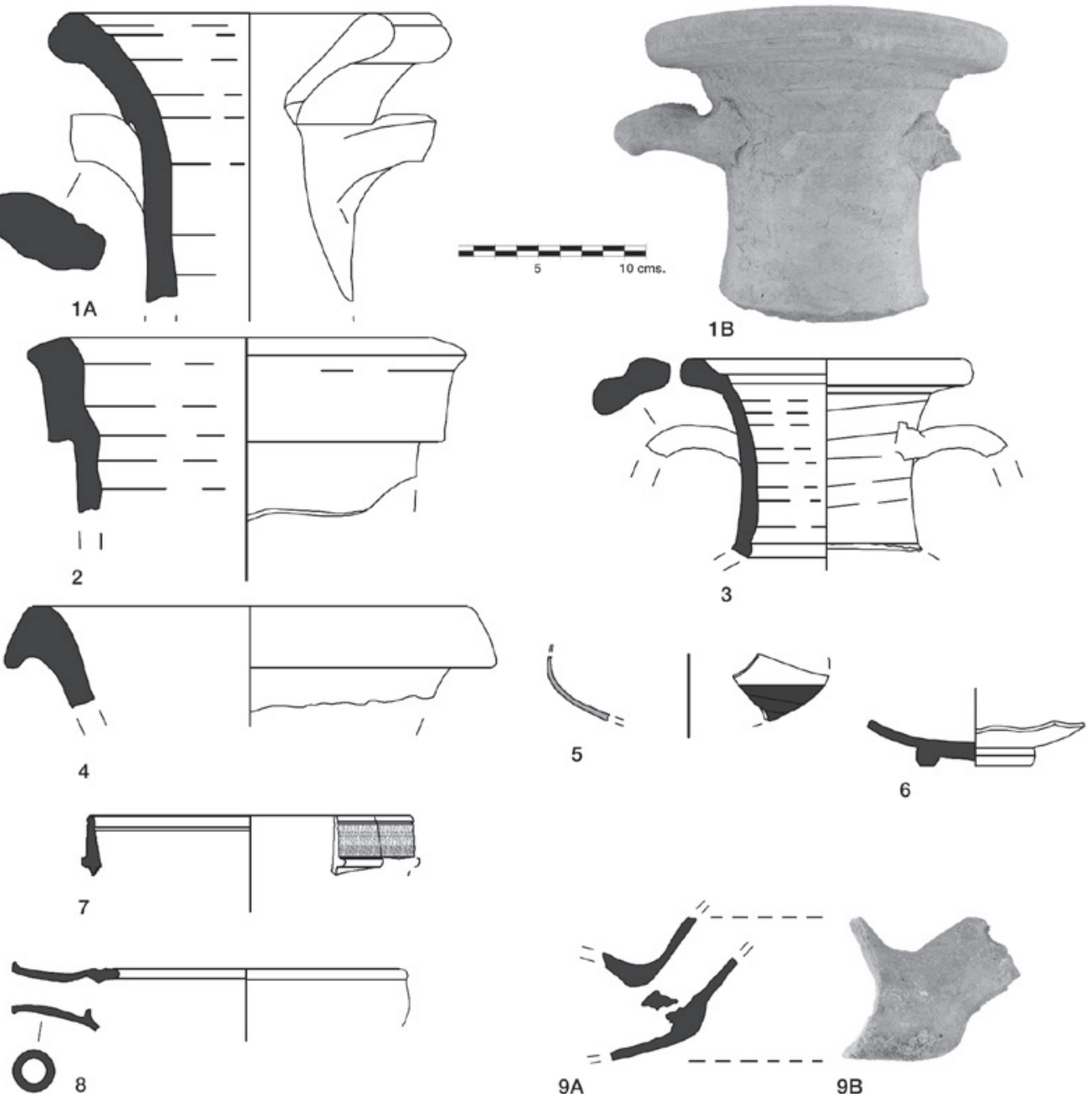

$9 \mathrm{~A}$

9B

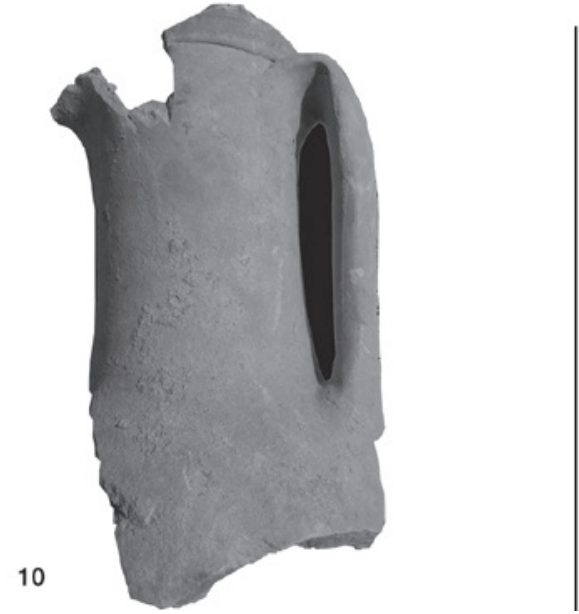

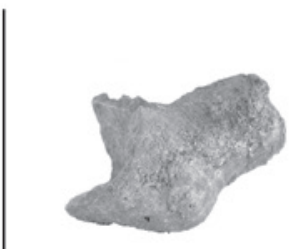

11

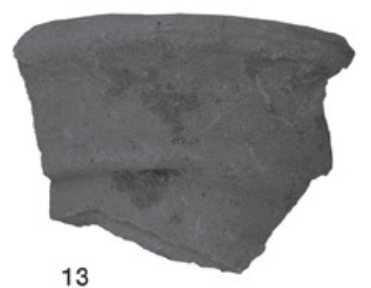

13

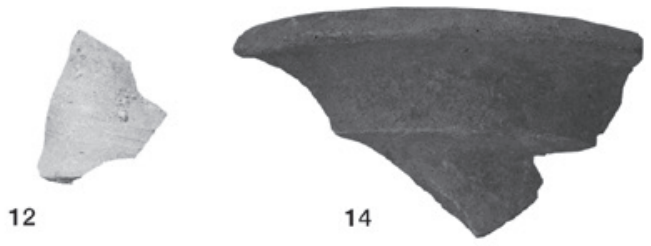

Figura 4.- Selección de cerámicas aparecidas en C/ Solano. Relleno interior del horno: ánforas Dr. 7/11 (1-2), G-4 (3) y Beltrán IIa (4); cerámica de paredes finas (5); fondo de T.S.H. (6), borde de Drag. 24/25 en T.S.G. (7); forma abierta de cerámica común (8) y askos (9). Estrato de cubrición del horno: ánfora Dr. 7/11 (10). Defectos de cocción: galbos de askos (11) y de cerámica común (12); ánforas Dr. 7/11 hipercocidas (13-14). 
material fabricado en el alfar también se documentaron cerámicas de importación que han permitido fechar el momento de cese de la actividad del mismo. Así, en el mencionado relleno aparecieron como material cerámico datante un borde de una copa de la forma Drag. 24/25 de TSG, así como un galbo de cerámica de paredes finas, el fondo de una forma abierta de TSH y un ánfora vinaria gálica de fondo plano (Gauloise 4), así como varias ánforas altoimperiales del tipo Dr. 20.

En resumen, podemos afirmar que el alfar de la $\mathrm{C} /$ Solano, que iniciaría su producción en un momento aún indeterminado de la primera mitad del s. I d.C. debió cesar su actividad en la segunda mitad de esa centuria (quizás en época flavia inicial, por la ausencia de ARSW A). Sin embargo, el hábitat en el barrio industrial de Gades continuaría, ahora más concentrado, durante algunas centurias más, tal y como demuestra la pervivencia de la actividad salazonera en la factoría de Teatro Andalucía. Por su parte, en el solar de la C/ Solano se extendería en momentos posteriores al cese de la actividad alfarera un área de necrópolis de inhumación datada genéricamente entre los ss. III y IV d.C. Y colmatando estas tumbas se localizaron una serie de paquetes deposicionales con abundantes $\mathrm{si}$ gillatas africanas (ARSW D, formas Hayes 59, 69 y 91), así como ánforas africanas (Keay XLI y XXV), sudhispánicas (Keay XIX, Almagro 51c) y orientales (Keay LIII) que denotan una intensa actividad comercial en la zona durante la segunda mitad del s. IV y parte del s. V d.C.

\section{BIBLIOGRAFÍA}

BERNAL CASASOLA, D. (2008): “Gades y su bahía en la Antigüedad. Reflexiones geoarqueológicas y asignaturas pendientes", en O. Arteaga y H.D. Schultz (eds.), Geoarqueología y proceso histórico en la Bahía de Cádiz, Revista Atlántica-Mediterránea de Prehistoria y Arqueología Social 10: 267-308.

BLANCO JIMÉNEZ, F.J. (1991): “Excavaciones de urgencia en un solar de la Calle Gregorio Marañón. Cádiz”. Anuario Arqueológico de Andalucía 1989, Tomo III: 78-81. Sevilla.

BLANCO JIMÉNEZ, F.J. y ALARCÓN CASTELLANO, F.J. (1996): Informe arqueológico: Avance de los resultados obtenidos en la I.A.U. realizada en la C/Sagasta $n^{\circ} 28$ (Cádiz), ejemplar inédito depositado en la Delegación Provincial de Cultura de la Junta de Andalucía en Cádiz.

COBOS, L.; MUÑOZ, A. y PERDIGONES, L. (1997): "Intervención arqueológica en el solar del antiguo Teatro Andalucía de Cádiz: la factoría de salazones y la representación gráfica del faro de Gades", Boletín del Museo de Cádiz 7: 115-132.

EXPÓSITO ÁLVAREZ, J.A. (2007): Las factorías de salazón de Gades (siglos II a.C.-VI d.C.). Estudio arqueológico y estado de la cuestión. Universidad de Cádiz.

GARCÍA VARGAS, E. (1998): La producción de ánforas en la Bahía de Cádiz en época romana (ss. II a.C.-IV d.C.). Écija.

FeChA DE ENTRADA: 19-12-2009

FECHA DE ACEPTACIÓN: 3-12-2010 